\title{
Elective lung resection increases spatial QRS-T angle and QTc interval
}

\author{
Szymon Bialka ${ }^{1}$, Andrzej Jaroszynski ${ }^{2}$, Todd T. Schlegel ${ }^{3,4}$, Hanna Misiolek ${ }^{1}$, \\ Damian Czyzewski ${ }^{5}$, Marek Sawicki ${ }^{6}$, Piotr Skoczylas ${ }^{6}$, Magdalena Bielacz ${ }^{7}$, \\ Mateusz Bialy ${ }^{8}$, Lukasz Szarpak ${ }^{9}$, Wojciech Dabrowski ${ }^{8}$
}

${ }^{1}$ Department of Anaesthesiology and Intensive Therapy, School of Medicine with Division of Dentistry in Zabrze, Medical University of Silesia, Katowice, Poland

${ }^{2}$ Department of Nephrology, Institute of Medical Science, Jan Kochanowski University of Kielce, Poland

${ }^{3}$ Department of Molecular Medicine and Surgery, Karolinska Institute, Stockholm, Sweden

${ }^{4}$ Nicollier-Schlegel SARL, Trélex, Switzerland

${ }^{5}$ Department of Thoracic Surgery, School of Medicine with Division of Dentistry in Zabrze, Medical University of Silesia, Katowice

${ }^{6}$ Department of Thoracic Surgery, Medical University of Lublin, Poland

${ }^{7}$ Institute of Tourism and Recreation, State Vocational College of Szymon Szymonowicz, Zamosc, Poland ${ }^{8}$ Department of Anaesthesiology and Intensive Therapy, Medical University of Lublin, Poland

${ }^{9}$ Lazarski University, Warsaw, Poland

\begin{abstract}
Background: Lung resection changes intra-thoracic anatomy, which may affect electrocardiographic results. While postoperative cardiac arrhythmias have been recognized after lung resection, no study has documented changes in vectorcardiographic variables in patients undergoing this surgery. The purpose of this study was to analyse changes in spatial QRS-T angle (spQRS-T) and corrected QT interval (QTc) after lung resection.

Methods: Adult patients undergoing elective lung resection under general anaesthesia were studied. The patients were allocated into four groups: those undergoing (1) left lobectomy $(L L)$; (2) left pneumonectomy (LP); (3) right lobectomy (RL); and (4) right pneumonectomy (RP). The spQRS-T angle and QTC interval were measured one day before surgery (baseline) and 24, 48 and $72 \mathrm{~h}$ after surgery.

Results: Seventy-one adult patients (47 men and 24 women) aged 47-80 (65 \pm 7) years were studied. In the study group as a whole, lung resection was associated with significant increases in spQRS-T $(p<0.001)$ and QTc $(p<0.05$ at 24 and $48 \mathrm{~h}$ and $p<0.01$ at $72 \mathrm{~h})$. The greatest changes were noted in patients undergoing LP. Postoperative atrial fibrillation $(A F)$ was noted in $6.4 \%$ of patients studied, in whom the widest spQRS-T angle and the most prolonged QTc intervals were also noted.

Conclusions: Lung resection widens the spQRS-T angle and prolongs the QTc interval, especially in patients undergoing $L P$. While postoperative $A F$ was a relatively rare complication after lung resection in this study, it was associated with the widest spQRS-T angles and most prolonged QTc intervals. (Cardiol J 2020; 27, 6: 705-714)
\end{abstract}

Key words: vectorcardiography, spatial QRS-T angle, corrected QT interval, atrial fibrillation, general anaesthesia

Address for correspondence: Wojciech Dabrowski, MD, PhD, Department of Anaesthesiology and Intensive Therapy, Medical University of Lublin, ul. Jaczewskiego 8, 20-954 Lublin, Poland, tel: +48 8172443 32,

e-mail: w.dabrowski5@yahoo.com

Received: 28.09.2018 Accepted: 28.11.2018 


\section{Introduction}

Cardiac arrhythmias, including atrial fibrillation (AF), have been recognized as frequent complications after thoracic surgery [1-4]. Different kinds of cardiac arrhythmias have been noted in one-fifth of patients after lung resection, $\mathrm{AF}$ being the most common $[4,5]$. Many studies have documented an increased risk of morbidity and mortality in thoracic surgical patients with postoperative cardiac arrhythmias [5-11]; however, only some have proposed risk factors for predicting postoperative cardiac disorders [9-11]. A history of ischemic heart disease, cardiovascular disorders and electrocardiographic $\mathrm{Q}$ waves are known predisposing factors for postoperative cardiac arrhythmias $[10,11]$.

Postoperative cardiopulmonary complications occur within 30 days after pulmonary resection and include pulmonary edema, ventricular fibrillation, complete heart block, various cardiac arrhythmias and sudden cardiac death $[10,11]$. Changes in intra-thoracic anatomy following lung resection may affect heart function, leading to hemodynamic and electrocardiographic disorders. Experimental studies have documented supraventricular tachycardia (sinus tachycardia and AF) occurring within 2 weeks after lobectomy or pneumonectomy [12]. Other electrocardiographic changes following pneumonectomy or lobectomy have included increases in P duration, ST-depression, delayed precordial R-wave transition and displacement of the $\mathrm{QRS}$ vector. The most striking changes were noted in patients who underwent left pneumonectomy or left upper segmentectomy and left lower lobectomy [7]. However, according to available research, no study has documented changes in spatial QRS-T angle (spQRS-T) or corrected QT interval (QTc) after lung resection.

Vectorcardiography (VCG) is a spatial method of electrocardiography (ECG) that enables visualizing, through the cardiac cycle, the continuous moments of the cardiac vector as loops. Vectorcardiograms and associated parameters such as the spQRS-T, i.e., the spatial angle between cardiac depolarization and repolarization, can also now be easily derived from standard 12-lead ECGs [13, 14]. While normal ranges for spQRS-T vary by method and by study, most studies have suggested that normal values lie below $100-110^{\circ}$ for men and below $90^{\circ}$ for women [14-17]. Of note, a widened spQRS-T is an independent predictor of cardiac arrhythmias and sudden cardiac death [14-17]. In healthy patients without apparent ECG pathol- ogy, cardiovascular mortality has been found to be $0.8 \%$ for spQRS-T between 0 and $50^{\circ}$, increasing to $2.3 \%$ for borderline spQRS-T $\left(50-100^{\circ}\right)$ and to $5.1 \%$ for spQRS-T angle between $100^{\circ}$ and $180^{\circ}$ $[15,17]$. Additionally, spQRS-T wider than $100^{\circ}$ can identify cardiomyopathic patients at a high risk of life-threatening ventricular arrhythmias for whom cardioverter-defibrillator devices might be most appropriate [18].

The corrected QT interval is an electrocardiographic parameter providing information on both cardiac depolarization and repolarization, especially the latter. Its prolongation has been observed in patients with electrolyte disorders and coronary diseases who are treated with antiarrhythmics or subjected to general anaesthesia with a volatile anesthetic [19-21]. A prolonged QTc interval has been well recognized as a parameter indicating an increased risk of malignant ventricular arrhythmias $[22,23]$. Although a prolonged QTc has been noted in patients undergoing surgery with pneumoperitoneum [24, 25] and cardiac surgery [26], changes in QTc have not been recognised in patients undergoing lobectomy or pneumonectomy. It should be noted that severe right and/or left ventricular dysfunction has been documented echographically following lobectomy or pneumonectomy [27]. The purpose of this study was to analyze changes in spQRS-T and QTc in patients undergoing lung resection.

\section{Methods}

\section{Ethical considerations}

This prospective observational study was conducted in adult patients undergoing elective thoracic surgery under general anesthesia. This study was conducted in accordance with the Declaration of Helsinki and was approved by the Institutional Review Board and the Bioethical Committee for Human Studies of the Medical University at Lublin, Poland (KE-0254/220/2016). Written informed consent was obtained from all participants. One day before surgery all patients were examined and a 12-lead ECG without overt clinical abnormality was the main criterion for patient inclusion. The exclusion criteria were: congenital heart defects, myocardial infarct and myocarditis history, cerebral stroke history, endocrine and metabolic diseases. Patients who required re-thoracotomy due to postoperative bleeding were also excluded.

\section{Patient description}

Anaesthesia. One day before surgery, routine blood examination was performed to assess 
complete blood count, blood glucose and urea, creatinine, serum electrolytes and blood type. All patients received the same premedication composed of a single oral dose $(2 \mathrm{mg})$ of estazolam (Polfa, Warsaw, Poland). Just before anaesthesia induction, all patients were routinely monitored with continuous ECG for heart rate, intermittent and non-invasive arterial blood pressure measurements and continuous pulse oximetry $\left(\mathrm{SpO}_{2}\right)$. The urinary bladder was catheterized in all patients before anaesthesia induction.

General anesthesia was induced intravenously with a single dose of fentanyl (Polfa, Warsaw, Poland) and propofol (AstraZeneca, United Kingdom). A single bolus of rocuronium $(0.6 \mathrm{mg} / \mathrm{kg}$ body weight) (Rocuronium B. Braun, Germany) was administered to each patient before tracheal intubation with a double-lumen tracheal tube, the placement of which was controlled with bronchoscopy. All participants were ventilated using intermittent positive pressure ventilation, and anaesthesia was maintained throughout the procedure using $100 \%$ oxygen and inhaled sevoflurane (AbbVie, UK) at a dose of 0.8-1.2 minimal alveolar concentration and fractionated doses of fentanyl. Intra-operative ventilation was performed with an expiratory/ inspiratory ratio of $1: 2$, a tidal volume of $5-6 \mathrm{~mL} /$ $/ \mathrm{kg}$ body weight, a respiration rate of $9-12 / \mathrm{min}$ and positive end-respiratory pressure (PEEP) $+5 \mathrm{~cm}$ $\mathrm{H}_{2} \mathrm{O}$. Ventilation was controlled by $\mathrm{EtCO}_{2}$ and $\mathrm{SpO}_{2}$, which were maintained between $35-40 \mathrm{mmHg}$ and $92-100 \%$ saturation during surgery with both double and single lung ventilation monitored via SpaceLabs monitor (SpaceLabs Healthcare, OSI systems, USA). During surgery, all patients received a continuous infusion of balanced crystalloids (Sterofundin ISO, B. Braun Melsungen, Germany). In patients not responding adequately to the crystalloid infusion, a single dose of ephedrine (Ephedrini hydrochlorici, Polfa Warsaw, Poland) was administered. Anaesthesia was used to maintain mean arterial pressure above $70 \mathrm{mmHg}$. After completion of the surgery, sevoflurane was discontinued. The neuromuscular blockade was reversed using a single dose of atropine $(0.5 \mathrm{mg})$ and neostigmine $(2.5 \mathrm{mg})$. Patients were extubated upon satisfactory emergence from general anesthesia and admitted to the Postoperative Intensive Care Unit. Postoperative pain was treated with fractionated doses of paracetamol (Bristol Myers Squibb, Warsaw, Poland). During the first and second postoperative days, all patients received intravenous mixture of potassium and magnesium at doses that depended on blood concentrations.
Surgery. After induction of anesthesia, pulmonectomy or lobectomy was performed with thoracotomy through the $4^{\text {th }}$ or $5^{\text {th }}$ intercostal space. Then, pleuraecotomy was performed through the $6^{\text {th }}$ intercostal space. The chest was closed in layers, including muscle, subcutaneous tissue, and the subcuticular layer, using resorbable sutures. Skin staples were used for the final cutaneous suture (Johnson and Johnson, New Jersey, USA).

\section{Measurements}

ECG, derived VCG. The method used for VCG measurement was presented previously [23]. Surface 12-lead resting ECG was recorded using a Cardiax device (IMED Co., Ltd., Budapest, Hungary). The recordings in each time period were automatically averaged to a single beat and transformed into three orthogonal leads, $\mathrm{X}, \mathrm{Y}$ and $\mathrm{Z}$, according to the inverse Dower method [14, 28]. The projections of maximum vectors of $\mathrm{QRS}$ and $T$-waves in frontal, transverse, and left sagittal planes and on $\mathrm{x}, \mathrm{y}$, and $\mathrm{z}$ axes were then obtained. Next, the value for the spatial QRS-T angle automatically calculated from the maximum spatial QRS and T vectors, as well as the values for the QTc interval, were obtained directly from Cardiax software. For detection of the QT interval and calculation of QTc, Cardiax software utilizes a medianbeats related "global QT interval" algorithm similar to that described by Xue [29].

Study protocol and patient division. Electrocardiography was performed at four time points: 1 day before surgery (baseline value) and 24, 48 and $72 \mathrm{~h}$ after surgery. All measurements were performed in the horizontal position. Patients were allocated into four groups: patients undergoing left pneumonectomy (LP), left lobectomy (LL), right pneumonectomy (RP) or right lobectomy (RL), respectively.

\section{Statistical analysis}

A statistical analysis was performed using Statistica 12 software (StatSoft). Demographic data were summarized as frequencies and percentages. Categorical variables were compared using the $\chi^{2}$ and Fisher exact test or $\chi^{2}$ with the Yates correction where applicable. For variables with a normal distribution, means and standard deviations (SD) were calculated, and the Student unpaired $t$-test was used for variables with a normal distribution as confirmed by the Shapiro-Wilk test. For variables with a non-normal distribution, the Wilcoxon signed-rank, Mann-Whitney-U, Kruskal-Wallis ANOVA and post hoc Dunnett's multiple compari- 


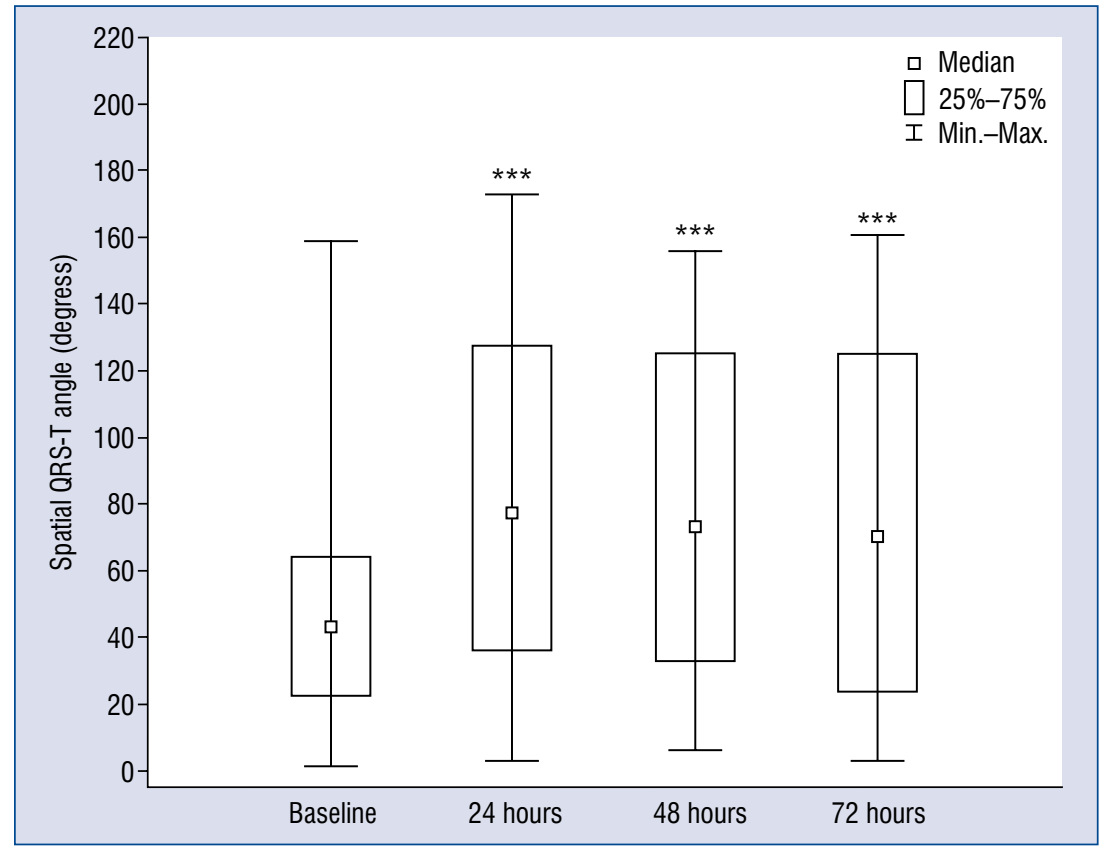

Figure 1. Changes in spatial QRS-T angle in the studied population. Time points: baseline -1 day before surgery, and 24,48 and 72 hours after surgery; ${ }^{* *} p<0.001$ in comparison with baseline.

son tests were used. A p $<0.05$ was considered significant. The power of the statistical tests was assessed by the $\mathrm{G}^{*}$ Power test.

\section{Results}

Seventy-one adult patients (47 men and 24 women) aged $65 \pm 7$ years (range 47-80), undergoing general anaesthesia and elective lung surgery for pulmonary neoplasm were included. In the studied group: 32 patients underwent left lobectomy (group LL: 14 patients - upper lobectomy and 18 patients - lower lobectomy), 9 - left pneumonectomy (group LP), 23 - right lobectomy (group RL: 10 patients - upper lobectomy and 13 patients - lower lobectomy) and 7 - right pneumonectomy (group RP). Forty-nine patients were treated for concomitant arterial hypertension ( $\mathrm{I}^{\circ}$ or $\mathrm{II}^{\circ}$ according to the World Health Organization classification) with beta-blockers (38 patients), $\mathrm{Ca}^{+2}$ blockers (9 patients), angiotensin converting enzyme inhibitors (44 patients) and diuretic drugs. Twenty-six patients were prescribed magnesium and 10 both magnesium and potassium for more than 1 month prior to surgery. Among all participants, the mean time of anaesthesia was $161 \pm 45$ min, and the surgical time was $115 \pm 30.7 \mathrm{~min}$. The mean body mass index was $26.6 \pm 5.2 \mathrm{~kg} / \mathrm{m}^{2}$. The mean arterial pressure and $\mathrm{SpO}_{2}$ were $68-100$ $\mathrm{mmHg}$ and $92-100 \%$, respectively, during anaes- thesia and surgery. None of the patients required intensive fluid therapy during surgery or the early postoperative period, and a possible insufficiency of intravascular fluids was supplemented with crystalloid infusion (Sterofundin ISO, B. Braun Melsungen, Germany). Immediately after anaesthesia completion, all patients were transferred to the Postoperative Intensive Care Unit. A sinus rhythm was noted in all participants with mean heart rates of $76.4 \pm 15.7 / \mathrm{min}, 78.5 \pm 14.8 / \mathrm{min}$ and $81.5 \pm 14.1 / \mathrm{min}$ at postoperative days 1,2 and 3 , respectively.

Atrial fibrillation was noted in $9(6.4 \%)$ patients at 5-10 postoperative days -7 after left lung surgery and 2 after right lung surgery $\left(\chi^{2}=1.69\right.$, $\mathrm{p}=0.19, \chi^{2}$ with Yates correction $=0.89$ for $\mathrm{p}=0.35$ ). Additionally, postoperative $\mathrm{AF}$ was noted in 6 patients after pneumonectomy (LP) and 3 after lobectomy ( 1 after LL and 2 after RL) $\left(\chi^{2}=11.5\right.$, $\mathrm{p}=0.001, \chi^{2}$ with Yates correction $=8.79$ for $\mathrm{p}=0.01)$. None of the other patients required treatment for postoperative cardiac arrhythmias, and the 12-lead ECG showed a sinus rhythm throughout the study period.

In the patient group as a whole, spQRS-T increased at 24, 48 and $72 \mathrm{~h}$ after surgery (Fig. 1). Changes in spQRS-T were similar in patients undergoing left and right lung surgery; however, a significantly wider spQRS-T was noted in patients after pneumonectomy than lobectomy (Table 1). 
Table 1. Comparison of changes in spatial QRS-T angle and QTc intervals in patients undergoing left lobectomy (group LL) pneumonectomy (group LP), right lobectomy (group RL) and right pneumonectomy (group RP).

\begin{tabular}{|c|c|c|c|c|}
\hline & Baseline & 24 hours & 48 hours & 72 hours \\
\hline \multicolumn{5}{|c|}{ Spatial QRS-T angle } \\
\hline LL & $\begin{array}{c}38.5 \\
{[31 ; 56]}\end{array}$ & $\begin{array}{c}80^{* *} \\
{[63.25 ; 93.5]}\end{array}$ & $\begin{array}{c}70.5^{*} \\
{[48 ; 87]}\end{array}$ & $\begin{array}{c}67.5 \\
{[21.25 ; 115.5]}\end{array}$ \\
\hline LP & $\begin{array}{c}56 \ddagger \\
{[50.25 ; 84.25]}\end{array}$ & $\begin{array}{c}129 * \text { } \\
{[128 ; 133.5]}\end{array}$ & $\begin{array}{c}147 * \text { †† } \\
{[130.5 ; 149.25]}\end{array}$ & $\begin{array}{c}145^{*} \text { ††キキ } \\
{[134.5 ; 154]}\end{array}$ \\
\hline $\mathrm{RL}$ & $\begin{array}{c}47.5+\dagger \\
{[24.75 ; 97.75]}\end{array}$ & $\begin{array}{c}47 \\
{[31.25 ; 135]}\end{array}$ & $\begin{array}{c}72 * \\
{[38.25 ; 137]}\end{array}$ & $\begin{array}{c}65^{*} \\
{[30.5 ; 120.75]}\end{array}$ \\
\hline $\mathrm{RP}$ & $\begin{array}{c}20 \\
{[6 ; 27]}\end{array}$ & $\begin{array}{c}33 \\
{[10.5 ; 47]}\end{array}$ & $\begin{array}{c}42^{*} \\
{[9.5 ; 99.5]}\end{array}$ & $\begin{array}{c}43^{*} \\
{[15 ; 109.5]}\end{array}$ \\
\hline \multicolumn{5}{|c|}{ Corrected QT interval } \\
\hline LL & $\begin{array}{c}425.5 \\
{[420 ; 442.2]}\end{array}$ & $\begin{array}{c}439.5 \\
{[429.5 ; 452.75]}\end{array}$ & $\begin{array}{c}435 \\
{[420.5 ; 558]}\end{array}$ & $\begin{array}{c}446^{* *} \\
{[430.5 ; 455.75]}\end{array}$ \\
\hline LP & $\begin{array}{c}443.5 \ddagger \\
{[59.25 ; 85]}\end{array}$ & $\begin{array}{c}462.5 \neq \ddagger \\
{[98 ; 147]}\end{array}$ & $\begin{array}{c}464.5 \\
{[111.5 ; 156.3]}\end{array}$ & $\begin{array}{c}462^{*} \\
{[93.6 ; 174.5]}\end{array}$ \\
\hline $\mathrm{RL}$ & $\begin{array}{c}428 \\
{[418.5 ; 478]}\end{array}$ & $\begin{array}{c}434 \\
{[421 ; 465.25]}\end{array}$ & $\begin{array}{c}431.5 \\
{[407.5 ; 480]}\end{array}$ & $\begin{array}{c}444.5 \\
{[427.75 ; 480.75]}\end{array}$ \\
\hline $\mathrm{RP}$ & $\begin{array}{c}411 \\
{[406 ; 414]}\end{array}$ & $\begin{array}{c}421 \\
{[412 ; 423]}\end{array}$ & $\begin{array}{c}414 \\
{[409 ; 443]}\end{array}$ & $\begin{array}{c}406 \\
{[403 ; 423]}\end{array}$ \\
\hline
\end{tabular}

Values shown are medians [quartile 1 and 3]. $L L(n=32), L P(n=9), R L(n=23), R P(n=7)$. The small number of patients with pneumonectomy limits statistical power $(1-\beta \geq 0.8)$.

${ }^{*} p<0.05$ versus Baseline; $\dagger p<0.05$ and $\dagger+p<0.01-$ pneumonectomy versus lobectomy (LP vs. LL and RP vs. RL, respectively); $\neq p<0.05$ and $\neq \neq p<0.01$ - left versus right pneumonectomy or left versus right lobectomy (LP vs. RP and LL vs. RL, respectively).

Table 2. Comparison of changes in spatial QRS-T angle and QTc intervals in patients with atrial fibrillation $(\mathrm{AF})$ in the late postoperative period and patients with an uncomplicated postoperative period (n-AF).

\begin{tabular}{lcccc}
\hline & Baseline & 24 hours & 48 hours & 72 hours \\
\hline Spatial QRS-T angle & & & & 102 \\
AF & 50 & $129^{*}+$ & $143^{*}$ & {$[59 ; 145]$} \\
n-AF & {$[47 ; 56]$} & {$[88 ; 135.5]$} & {$[75 ; 147]$} & $66^{* *}$ \\
Corrected OT interval & 33.5 & $69 * *$ & $71.5^{* *}$ & {$[24 . ; 123.5]$} \\
AF & {$[20.25 ; 68.75]$} & {$[32.25 ; 116.75]$} & {$[26.25 ; 120.75]$} & $462^{*}$ \\
& & & $469 \dagger$ & {$[451.5 ; 467]$} \\
n-AF & 439 & 453 & {$[455 ; 470.5]$} & 435 \\
& {$[427.5 ; 445.5]$} & {$[439 ; 462.75]$} & 434.5 & {$[423 ; 455.75]$} \\
\hline
\end{tabular}

Values shown are medians [quartile 1 and 3]. AF $(n=9), n-A F(n=62)$. The small number of patients with postoperative AF limits statistical power $(1-\beta \geq 0.8)$.

${ }^{*} \mathrm{p}<0.05$ and ${ }^{* *} \mathrm{p}<0.001$ versus Baseline; $\mathrm{\dagger p}<0.05$ - complicated postoperative period by AF versus uncomplicated postoperative period

Additionally, a wider spQRS-T was noted after LP than RP at all time points whereas this angle was comparable in patients undergoing LL and RL (Table 1). In patients with, compared to those without postoperative AF, the spatial QRS-T angle was significantly wider only at $24 \mathrm{~h}$ postoperatively (Table 2). Representative serial 12-lead ECGs from a patient who underwent LP are presented in Figure 2.
In the patient group as a whole, QTc intervals were also prolonged after lung resection (Fig. 3). Although prolongation of the QTc was similar in patients undergoing left and right lung surgery independent of the type of surgery, LP prolonged the QTc significantly more than RP (Table 1 ). The corrected QT interval was also significantly longer at 48 and $72 \mathrm{~h}$ in patients with versus those without postoperative $\mathrm{AF}$ (Table 2 ). 


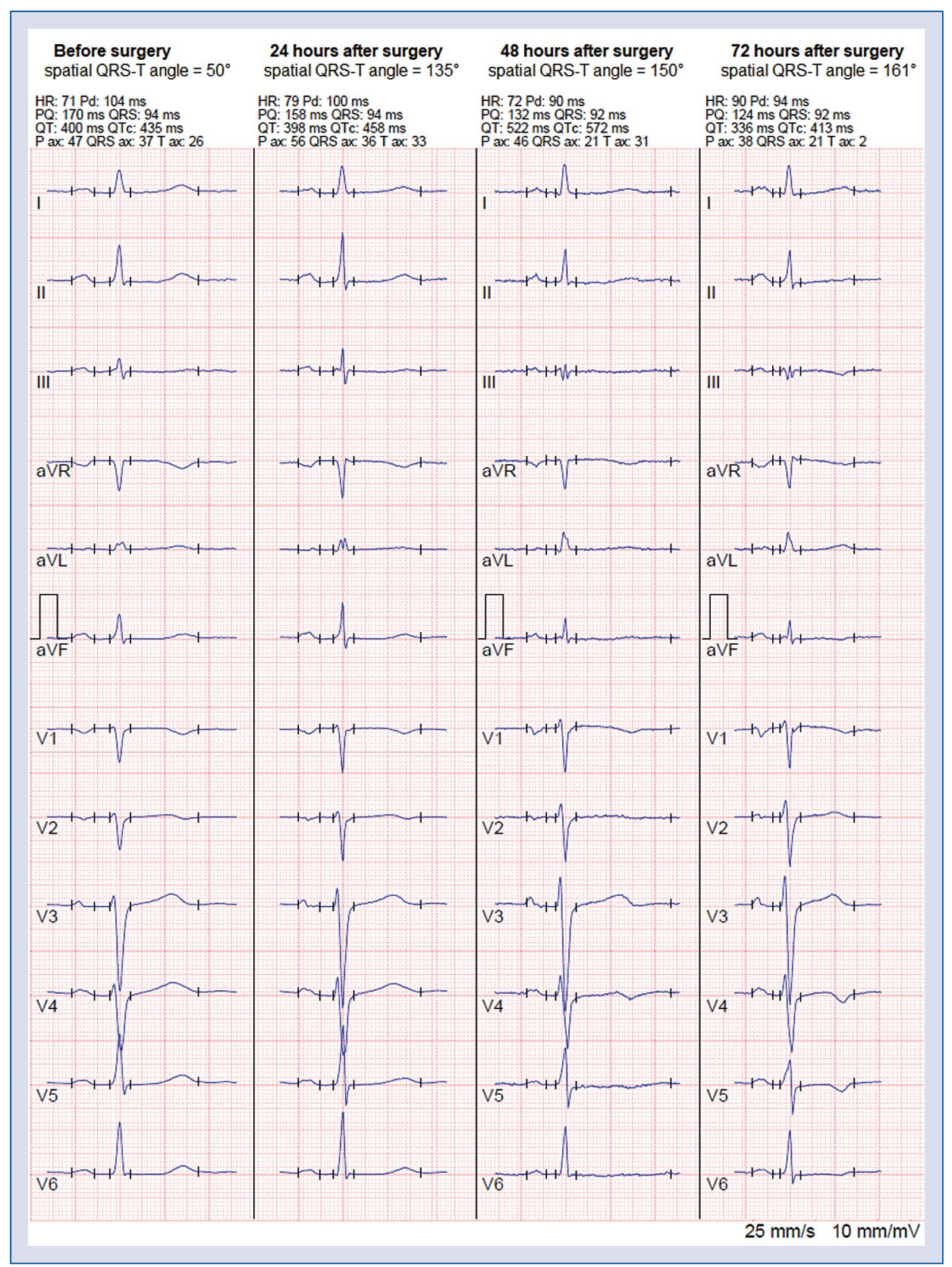

Figure 2. Representative changes in the spatial QRS-T angle and 12-lead electrocardiogram of a patient who underwent left pneumonectomy.

\section{Discu/ssion}

According to available research, this is the first study documenting effects of lung resection on spQRS-T and QTc. Pneumonectomy and lobectomy affected spQRS-T and QTc; however, the most striking changes were noted in patients undergoing left rather than right lung surgery, especially LP. Additionally, patients with postoperative AF had wider spQRS-T and longer QTc in the early postoperative period.
The spQRS-T angle presents the directional differences between the ventricular repolarization and depolarization [30]. It has been suggested to be a strong predictor for all-cause and cardiac mortality in middle-aged and elderly patients [15, 17, 30]. Additionally, a wide spQRS-T has been shown to predict life-threatening ventricular arrhythmias [15-18] and silent myocardial ischemia/ /infarction [31, 32]. Several factors affect spQRS-T. The spQRS-T angle evolves with age and depends 


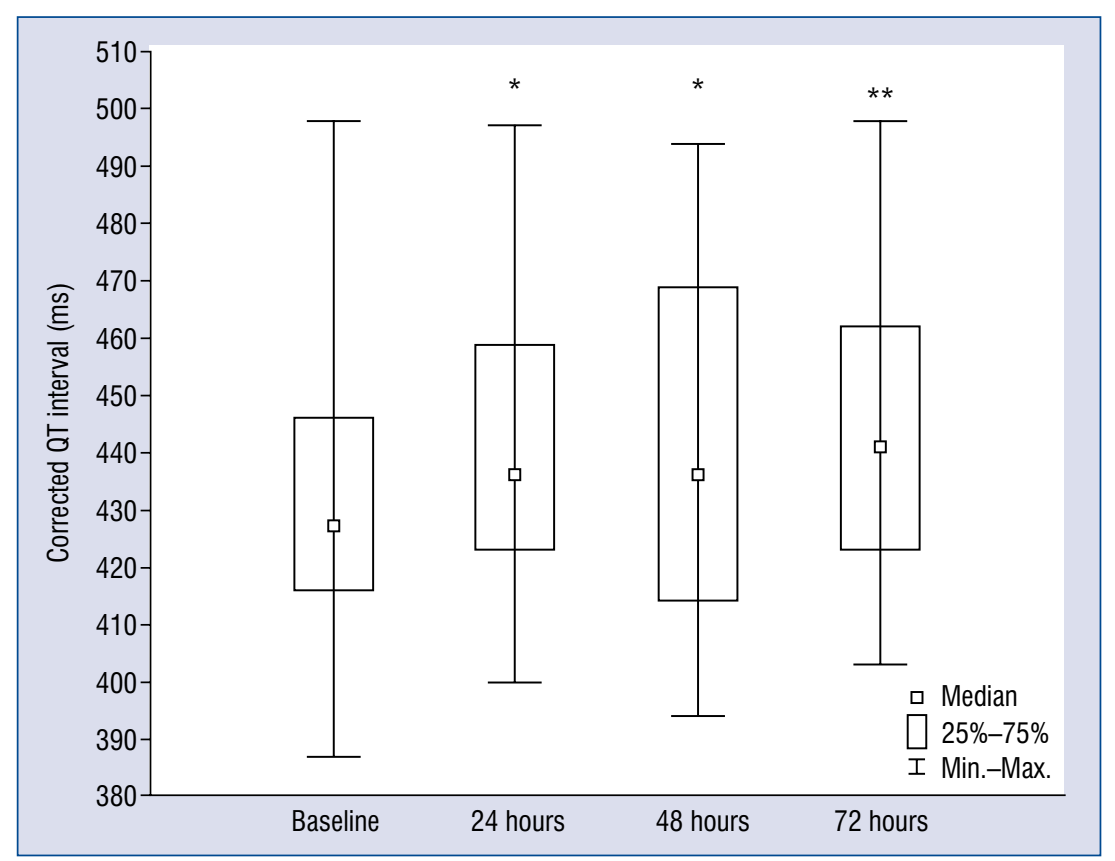

Figure 3. Changes in the corrected OT interval of the studied population. Time points: baseline -1 day before surgery, and 24, 48 and 72 hours after surgery; ${ }^{*} p<0.05$ and ${ }^{* *} p<0.01$ in comparison with baseline.

on sex and physical condition [13-17, 33, 34]. Changes in body position as well as in intra-thoracic volume following diaphragm elevation due to increases in intra-abdominal pressure, which may also disturb ventricular repolarization, thus widening the spQRS-T [25, 35, 36]. Gul and colleagues documented a completely altered QRS complex in patients with changes in intra-thoracic anatomy following eventration of the diaphragm and displacement of the bowels into the thoracic cavity [37].

In the present study, an increase in spQRS-T in patients after lung resection was observed, the most substantive changes occurring in patients after LP. A lack of or significant reduction in heart compression during respiration probably disturbs the QRS loop configuration and affects the heart's hemodynamic function. In people with normal pulmonary function, the amplitude of the maximum leftward force affects the QRS vector, decreasing significantly during inspiration, whereas the amplitude of the maximum posterior force increases. The QRS vector displaces posteriorly and inferiorly with a decrease in spatial magnitude of approximately $10 \%$, leading to an increase in the spQRS-T angle [37]. Previous studies have shown that rightlung resection causes a right-sided QRS vector shift in $100 \%$ of patients after RP and in $75 \%, 58.3 \%$ and $50 \%$ after right upper segmentectomy, lower left lobectomy and right upper segmentectomy, respectively [7]. On the other hand, left-lung resection results in a left-sided QRS vector shift in $100 \%$ of patients after LP and in 87\%, 66.7\%, 57.9\% and $55.6 \%$ of patients after left upper lobectomy, left upper segmentectomy, right lower lobectomy and right middle lobectomy, respectively [7]. The present study was not focused on, and thus did not fully confirm these disturbances. However, LP spectacularly increased spQRS-T in the patient group as a whole, with disturbances in spQRS-T being significantly lower in patients after RP. It should be noted that the small number of patients with pneumonectomy limited the power of the analysis, and may have impacted results.

A $6.4 \%$ incidence of postoperative $\mathrm{AF}$ was lower than that in other reports [1-6]. A significantly wider spQRS-T angle was noted in all of these patients. Several studies have confirmed a strong relationship between a wider spQRS-T and the occurrence of potentially lethal arrhythmias in patients with left ventricular systolic dysfunction [15-17, 30, 38]. The risk of cardiac arrhythmia significantly increases with spQRS-T wider than $100^{\circ}[15,16,30]$. It should be noted that spQRS-T increased above $100^{\circ}$ in relatively more patients with postoperative $\mathrm{AF}$ and that the higher angle was observed $48 \mathrm{~h}$ after surgery.

Postoperative AF might result from postoperative electrolyte disorders; however, all affected 
patients received potassium and magnesium supplementation, and their blood potassium concentration was higher than $4.0 \mathrm{mmol} / \mathrm{L}$ (data not shown). The incidence of $\mathrm{AF}$ was significantly higher in patients after pneumonectomy, in agreement with other studies [4-6]. Although none of the patients presented with myocardial ischemia, it can also be speculated that postoperative $\mathrm{AF}$ might be a result of silent myocardial ischemia. Many patients had a history of smoking and chronic obstructive pulmonary disease, which are often associated with atherosclerosis and coronary diseases. Indeed, silent myocardial ischemia/infarct has been shown to be associated with a widened spQRS-T angle [32]; however, this speculation would require further dedicated confirmation.

In the present study, QTc was prolonged after left-side resection and was significantly longer than after right-side resection. Additionally, QTc was longer in patients with postoperative AF. The QTc interval represents myocardial depolarization and repolarization. Its normal value is below $460 \mathrm{~ms}$ for women and below $440 \mathrm{~ms}$ for men, with borderline values ranging $461-479 \mathrm{~ms}$ and $441-459 \mathrm{~ms}$ for women and men, respectively; a prolonged value is defined as a value higher than $480 \mathrm{~ms}$ and 460 ms for women and men, respectively [23].

A prolonged QTc interval is also associated with an increased risk of life-threatening arrhythmias, including polymorphic ventricular tachycardia (torsade de points), and a significant increase in the risk for such pathology increases markedly in patients with QTc intervals longer than $500 \mathrm{~ms}$. However, the risk of cardiac arrhythmia also increases when QTc lengthens more than $60 \mathrm{~ms}$ compared with the baseline value [39].

Female sex and older age predisposes QTc interval prolongation. Pathologically prolonged QTc has been noted in patients with electrolyte disturbances such as hypokalemia, hypomagnesemia and/or hypocalcemia, myocardial ischemia, heart failure and severe bradycardia [19, 20, 31]. Many medications can also result in asymptomatic or symptomatic QTc interval prolongation, their administration sometimes being associated with an increased risk for cardiac arrhythmias [22].

It should be noted that volatile anaesthetics and some opioids, including fentanyl, significantly prolong QTc. However, there is controversy as to whether propofol has an effect [22, 40-42]. Chang et al. [41] documented a prolongation of QTc following propofol anesthesia. Oji et al. [40] noted the shortening of QTc after target-controlled infusion of propofol. In contrast, Kleinsasser et al. [42] did not observe an effect of propofol on QTc interval. Additionally, some local anaesthetics used for epidural anaesthesia during lung surgery can also affect QTc [9]. In the present study, a prolonged QTc only in patients undergoing left-lung resection was observed, but all participants received the same kind of anesthesia. Unfortunately for reasons of logistics, it was not possible to study changes in QTc more immediately after surgery.

\section{Limitations of the study}

An important limitation of the present study was the small number of patients undergoing elective pneumonectomy, which notably limits studyrelated statistical power. Generally, the available screening examinations enable cancer diagnosis in its early stages and have a fundamental role in the possible kinds of treatment. The lack of an analysis of spQRS-T and QTc as a function of blood electrolyte concentration or preoperative or postoperative spirometric features or pharmacological treatment was also considered a limitation.

\section{Conclusions}

In the present study, an effect of lung resection on spQRS-T and the QTc interval was documented. Generally, lung resection induced spQRS-T widening and QTc prolongation; however, the most striking widening and prolongation were noted in patients undergoing LP. Additionally, AF episodes were primarily noted in patients after left-lung resection who had prolonged QTc interval.

\section{Conflict of interest: None declared}

\section{References}

1. Giambrone GP, Wu X, Gaber-Baylis LK, et al. Incidence and implications of postoperative supraventricular tachycardia after pulmonary lobectomy. J Thorac Cardiovasc Surg. 2016; 151(4): 982-988, doi: 10.1016/j.jtcvs.2015.11.057, indexed in Pubmed: 26778376.

2. Sanecka A, Biernacka EK, Szperl M, et al. QTc prolongation in patients with hearing loss: Electrocardiographic and genetic study. Cardiol J. 2016; 23(1): 34-41, doi: 10.5603/CJ.a2015.0062, indexed in Pubmed: 26412604.

3. Lee JH, Lim C, Kim JS, et al. Early and mid-term results of coronary endarterectomy: Influence of cardiopulmonary bypass and surgical techniques. Cardiol J. 2017; 24(3): 242-249, doi: 10.5603/CJ.a2017.0027, indexed in Pubmed: 28281737.

4. Elrakhawy HM, Alassal MA, Elsadeck N, et al. Predictive factors of supraventricular arrhythmias after noncardiac thoracic surgery: a multicenter study. Heart Surg Forum. 2014; 17(6): E308-E312, doi: 10.1532/HSF98.2014412, indexed in Pubmed: 25586281. 
5. Iwata T, Nagato K, Nakajima T, et al. Risk factors predictive of atrial fibrillation after lung cancer surgery. Surg Today. 2016; 46(8): 877-886, doi: 10.1007/s00595-015-1258-4, indexed in Pubmed: 26471506.

6. Rena O, Papalia E, Oliaro A, et al. Supraventricular arrhythmias after resection surgery of the lung. Eur J Cardiothorac Surg. 2001; 20(4): 688-693, indexed in Pubmed: 11574209.

7. Chhabra L, Bajaj R, Chaubey VK, et al. Electrocardiographic impacts of lung resection. J Electrocardiol. 2013; 46(6): 697. e1-697.e8, doi: 10.1016/j.jelectrocard.2013.05.140, indexed in Pubmed: 23830322.

8. Ivanovic J, Maziak DE, Ramzan S, et al. Incidence, severity and perioperative risk factors for atrial fibrillation following pulmonary resection. Interact Cardiovasc Thorac Surg. 2014; 18(3): 340-346, doi: 10.1093/icvts/ivt520, indexed in Pubmed: 24336699.

9. Güven O, Sazak H, Alagöz A, et al. The effects of local anaesthetics on QT parameters during thoracic epidural anaesthesia combined with general anaesthesia: ropivacaine versus bupivacaine. Balkan Med J. 2013; 30(4): 410-414, doi: 10.5152/balkanmedj.2013.9275, indexed in Pubmed: 25207150.

10. Brunelli A, Cassivi SD, Fibla J, et al. External validation of the recalibrated thoracic revised cardiac risk index for predicting the risk of major cardiac complications after lung resection. Ann Thorac Surg. 2011; 92(2): 445-448, doi: 10.1016/j.athoracsur.2011.03.095, indexed in Pubmed: 21704295.

11. Miura N, Kohno M, Ito K, et al. Lung cancer surgery in patients aged 80 years or older: an analysis of risk factors, morbidity, and mortality. Gen Thorac Cardiovasc Surg. 2015; 63(7): 401-405, doi: 10.1007/s11748-015-0546-7, indexed in Pubmed: 25868520.

12. Kocatürk M, Salci H, Yilmaz Z, et al. Pre- and post-operative cardiac evaluation of dogs undergoing lobectomy and pneumonectomy. J Vet Sci. 2010; 11(3): 257-264, indexed in Pubmed: 20706034.

13. Draisma HHM, Schalij MJ, van der Wall EE, et al. Elucidation of the spatial ventricular gradient and its link with dispersion of repolarization. Heart Rhythm. 2006; 3(9): 1092-1099, doi: 10.1016/j.hrthm.2006.05.025, indexed in Pubmed: 16945809.

14. Cortez DL, Schlegel TT. When deriving the spatial QRS-T angle from the 12-lead electrocardiogram, which transform is more Frank: regression or inverse Dower? J Electrocardiol. 2010; 43(4): 302-309, doi: 10.1016/j.jelectrocard.2010.03.010, indexed in Pubmed: 20466388.

15. Yamazaki T, Froelicher VF, Myers J, et al. Spatial QRS-T angle predicts cardiac death in a clinical population. Heart Rhythm. 2005; 2(1): 73-78, doi: 10.1016/j.hrthm.2004.10.040, indexed in Pubmed: 15851268.

16. Borleffs CJ, Scherptong RWC, Man SC, et al. Predicting ventricular arrhythmias in patients with ischemic heart disease: clinical application of the ECG-derived QRS-T angle. Circ Arrhythm Electrophysiol. 2009; 2(5): 548-554, doi: 10.1161/CIRCEP.109.859108, indexed in Pubmed: 19843923.

17. Voulgari C, Pagoni S, Tesfaye S, et al. The spatial QRS-T angle: implications in clinical practice. Curr Cardiol Rev. 2013; 9(3): 197-210, indexed in Pubmed: 23909632.

18. Borleffs CJ, Scherptong RWC, Man SC, et al. Predicting ventricular arrhythmias in patients with ischemic heart disease: clinical application of the ECG-derived QRS-T angle. Circ Arrhythm Electrophysiol. 2009; 2(5): 548-554, doi: 10.1161/CIRCEP.109.859108, indexed in Pubmed: 19843923.

19. Michishita R, Ishikawa-Takata K, Yoshimura E, et al. Influence of dietary sodium and potassium intake on the heart rate cor-
rected-QT interval in elderly subjects. J Nutr Sci Vitaminol. 2015; 61(2): 138-146, doi: 10.3177/jnsv.61.138, indexed in Pubmed: 26052144 .

20. Thomas SHL, Behr ER. Pharmacological treatment of acquired QT prolongation and torsades de pointes. Br J Clin Pharmacol. 2016; 81(3): 420-427, doi: 10.1111/bcp.12726, indexed in Pubmed: 26183037.

21. Paventi S, Santevecchi A, Ranieri R. Effects of sevoflurane versus propofol on QT interval. Minerva Anestesiol. 2001; 67(9): 637-640, indexed in Pubmed: 11731753.

22. De Vecchis R, Ariano C, Di Biase G, et al. Malignant ventricular arrhythmias resulting from drug-induced QTc prolongation: a retrospective study. J Clin Med Res. 2018; 10(7): 593-600, doi: 10.14740/jocmr3470w, indexed in Pubmed: 29904445.

23. Steinberg C. Diagnosis and clinical management of long-QT syndrome. Curr Opin Cardiol. 2018; 33(1): 31-41, doi: 10.1097/ hco.0000000000000465.

24. Ekici Y, Bozbas H, Karakayali F, et al. Effect of different intraabdominal pressure levels on QT dispersion in patients undergoing laparoscopic cholecystectomy. Surg Endosc. 2009; 23(11): 2543-2549, doi: 10.1007/s00464-009-0388-4.

25. Dabrowski W, Jaroszynski A, Jaroszynska A, et al. Intra-abdominal hypertension increases spatial QRS-T angle and elevates ST-segment J-point in healthy women undergoing laparoscopic surgery. J Electrocardiol. 2017; 50(2): 214-222, doi: 10.1016/j. jelectrocard.2016.10.002, indexed in Pubmed: 28029353.

26. Mirbolouk F, Arami S, Salari A, et al. Corrected QT-interval and dispersion after revascularization by percutaneous coronary intervention and coronary artery bypass graft surgery in chronic ischemia. J Invasive Cardiol. 2014; 26(9): 444-450, indexed in Pubmed: 25198488.

27. Wang Z, Yuan J, Chu W, et al. Evaluation of left and right ventricular myocardial function after lung resection using speckle tracking echocardiography. Medicine. 2016; 95(31): e4290, doi: 10.1097/ MD.0000000000004290, indexed in Pubmed: 27495031.

28. Edenbrandt L, Pahlm O. Vectorcardiogram synthesized from a 12-lead ECG: superiority of the inverse Dower matrix. J Electrocardiol. 1988; 21(4): 361-367, indexed in Pubmed: 3241148.

29. Xue QT. Interval Measurement: What Can We Really Expect? Computers in Cardiology. 2006; 33(385): 388.

30. Kück K, Isaksen JL, Graff C, et al. Spatial QRS-T angle variants for prediction of all-cause mortality. J Electrocardiol. 2018; 51(5): 768-775, doi: 10.1016/j.jelectrocard.2018.05.011, indexed in Pubmed: 30177310.

31. Zhang ZM, Rautaharju PM, Prineas RJ, et al. Electrocardiographic QRS-T angle and the risk of incident silent myocardial infarction in the Atherosclerosis Risk in Communities study. J Electrocardiol. 2017; 50(5): 661-666, doi: 10.1016/j.jelectrocard.2017.05.001, indexed in Pubmed: 28515002.

32. Kamphuis V, Blom N, Zwet Ev, et al. Normal values of the ventricular gradient and QRS-T angle, derived from the pediatric electrocardiogram. J Electrocardiol. 2018; 51(3): 490-495, doi: 10.1016/j.jelectrocard.2018.01.002.

33. Kenttä T, Karsikas M, Kiviniemi A, et al. Dynamics and rate-dependence of the spatial angle between ventricular depolarization and repolarization wave fronts during exercise ECG. Ann Noninvasive Electrocardiol. 2010; 15(3): 264-275, doi: 10.1111/j.1542474X.2010.00374.x, indexed in Pubmed: 20645970.

34. Obata Y, Ruzankin P, Ong QiJ, et al. The impact of posture on the cardiac depolarization and repolarization phases of the QT interval in healthy subjects. J Electrocardiol. 2017; 50(5): 
640-645, doi: 10.1016/j.jelectrocard.2017.03.001, indexed in Pubmed: 28330682.

35. Dabrowski W, Schlegel TT, Wosko J, et al. Changes in spatial QRS-T angle and QTc interval in patients with traumatic brain injury with or without intra-abdominal hypertension. J Electrocardiol. 2018; 51(3): 499-507, doi: 10.1016/j.jelectrocard.2017.12.038, indexed in Pubmed: 29310923.

36. Gul EE, Can I, Ozbek O. Displacement of the heart by diaphragm: is this heart alternating? J Electrocardiol. 2011; 44(4): 465-466, doi: 10.1016/j.jelectrocard.2010.09.002, indexed in Pubmed: 21093872.

37. Yamada N. Effects of respiration on the vectorcardiogram obtained with the Frank lead system. Acta Med Okayama. 1985; 39(4): 297 -313, doi: 10.18926/AMO/31492, indexed in Pubmed: 4050536.

38. Pratt CM, Al-Khalidi HR, Brum JM, et al. Cumulative experience of azimilide-associated torsades de pointes ventricular tachycardia in the 19 clinical studies comprising the azimilide database. J Am Coll Cardiol. 2006; 48(3): 471-477, doi: 10.1016/j. jacc.2006.04.075, indexed in Pubmed: 16875971.

39. Muensterman ET, Tisdale JE. Predictive analytics for identification of patients at risk for QT interval prolongation: a systematic review. Pharmacotherapy. 2018; 38(8): 813-821, doi: 10.1002/ phar.2146, indexed in Pubmed: 29882591.

40. Oji M, Terao Y, Toyoda T, et al. Differential effects of propofol and sevoflurane on QT interval during anesthetic induction. J Clin Monit Comput. 2013; 27(3): 243-248, doi: 10.1007/s10877-0129420-7, indexed in Pubmed: 23242843.

41. Chang DJ, Kweon TD, Nam SB, et al. Effects of fentanyl pretreatment on the QTc interval during propofol induction. Anaesthesia. 2008; 63(10): 1056-1060, doi: 10.1111/j.1365-2044.2008.05559.x, indexed in Pubmed: 18616522.

42. Kuenszberg E, Loeckinger A, Kleinsasser A, et al. Sevoflurane, but not propofol, significantly prolongs the Q-T interval. Anesth Analg. 2000; 90(1): 25-27, indexed in Pubmed: 10624970. 